\title{
Structure and dynamics of the Shapley supercluster
}

\section{Dominique Proust ${ }^{1}$, Andreas Reisenegger ${ }^{2}$, Hernán Quintana $^{2}$,} Eric Slezak ${ }^{3}$, Hernán Muriel ${ }^{4}$, Laerte Sodré Jr. ${ }^{5}$, Michael J. Drinkwater ${ }^{6}$ and Quentin A. Parker ${ }^{7}$

${ }^{1}$ GEPI, Observatoire de Paris-Meudon F92195 Meudon CEDEX, France

${ }^{2}$ Departamento de Astronomìa y Astrofisica, Pontificia Universidad Católica de Chile, Casilla 306, Santiago 22, Chile

${ }^{3}$ Observatoire de Nice, 06304 Nice CEDEX4, France

${ }^{4}$ Grupo de Investigaciones en Astronomia Teórica y Experimental, Observatorio Astrónomico, Laprida 854, 5000 Córdoba, Argentina and CONICET, Buenos Aires, Argentina

${ }^{5}$ Instituto de Astronomia, Geofísica e Ciêncas Atmos., Universidade de São Paulo (IAG/USP), 01065-970 São Paulo/SP Brazil

${ }^{6}$ Department of Physics, University of Queensland, QLD 4072, Australia

${ }^{7}$ Department of Physics, Macquarie University, NSW 2109, Australia, and Anglo-Australian Observatory, PO Box 296, Epping NSW 1710, Australia

\begin{abstract}
We present results of our wide-field redshift survey of galaxies in a 285 square degree region of the Shapley Supercluster (SSC).
\end{abstract}

\section{Introduction}

The Shapley supercluster (SSC) is a remarkably rich concentration of galaxies centred around R.A. $=13^{h} 25^{m}$ Dec. $=-30^{\circ}$ which has been investigated by numerous authors since its discovery in 1930 (see Quintana et al. 1995). It consists of many clusters and groups of galaxies in the redshift range $0.04<z<0.055$, and lies in the general direction of the dipole anisotropy of the CMB. It is located $130 h^{-1} \mathrm{Mpc}$ beyond the HydraCentaurus supercluster. The SSC is one of the most massive concentrations of galaxies in the local universe, so it is also of particular interest to consider its effect on the dynamics of the Local Group. We present an analysis of the Shapley supercluster based on the most complete velocity catalogue obtained up to now. We discuss the completeness and overdensities of the velocity sample and the structure and dynamics of the supercluster using the spherical collapse model.

\section{The velocity catalogue}

We have made an investigation into the larger scale distributions of galaxies throughout the entire SSC region and close environs using data from wide-field multi-fibre spectrographs such as OPTOPUS and MEFOS at the 3.60m ESO telescope (Quintana et al. 1997), the fiber spectrograph at the 100" DuPont telescope at Las Campanas, Chile (Proust et al. 2004), the UKST/FLAIR-II system, Australia (Drinkwater et al. 1999, 2004), and more recently the UKST/6dF instrument (Jones et al. 2004). Combined with already published redshift sets from several surveys and papers (see Quintana et al. 1995, 2000, Bardelli et al. 2001, Kaldare et al. 2003) and data from the NED NASA/IPAC 


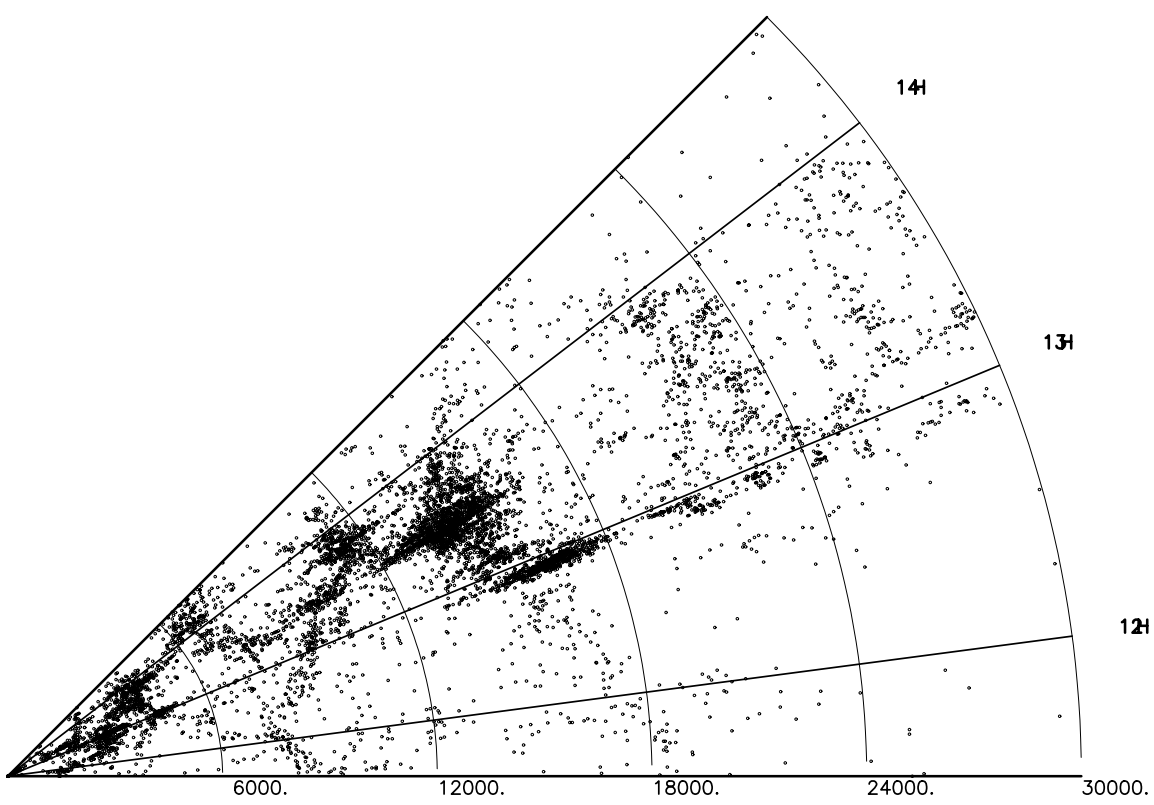

Figure 1. Wedge diagram in Right Ascension.

database, we obtain the most complete velocity catalogue for the SSC containing 10225 velocity measurements for 8341 galaxies (Figure 1). 4212 (50\%) galaxies belong to the SSC with velocities in the range $9000-18000 \mathrm{~km} \mathrm{~s}^{-1}$ (a total depth of $90 \mathrm{~h}^{-1} \mathrm{Mpc}$ ).

\section{Galaxy sample, completeness and overdensities}

We compare our sample of observed galaxies to the total magnitude-limited galaxy distribution in the 225 square degrees survey region. We use the SuperCOSMOS sky survey to construct the parent galaxy catalogue for the region and analyse $b_{j}$ magnitudes of all galaxies. Then, we determine the completeness of our survey (Drinkwater et al. 2004) as a function of 4 limiting magnitudes (Table 1) for both the full and the intercluster regions. It is highest for the brighter magnitude limits, peaking at $60 \%$ for the whole sample at $b_{j}<17.0$. In the velocity range $\left(9000<c z<18000 \mathrm{~km} \mathrm{~s}^{-1}\right)$ of the SSC, we calculate galaxy overdensities of $7.0 \pm 0.2$ over the 225 square degree central region and $4.8 \pm 0.2$ in a 192 square degree region excluding rich clusters. Over the large region of our survey the inter-cluster galaxies make up $48 \%$ (3705 objects) of all galaxies in the SSC region, and assuming a similar mass function they contribute a similar amount of mass to the cluster galaxies.

\section{Velocity dispersions of ACO clusters in the Shapley supercluster}

Velocity dispersions are an essential piece of information to study cluster dynamics, because they probe directly the cluster potential. To study the distribution of velocity dispersions of the Shapley clusters that are in the ACO catalog, we examined the velocity distribution of galaxies within 0.5 degree $(\sim$ Abell radius at $z=0.05$ with $h=0.7)$ of each cluster center in the ACO catalog. The number of clusters with at least 6 galaxies in our velocity sample is 46 . For a sample of 36 clusters in the best sampled region, the median velocity dispersion is $727 \mathrm{~km} \mathrm{~s}^{-1}$. All clusters in the $\mathrm{SSC}$ with velocity dispersion larger than $1000 \mathrm{~km} \mathrm{~s}^{-1}$ present evidence of substructures. 
Table 1. Completeness of the velocity catalogue for $4 b_{j}$ magnitude limits.

\begin{tabular}{lrrrl}
\hline Field & $b_{j}$ Mag lim. & Catalogue & Velocities & Differential \\
\hline Full & 17.0 & 2981 & $1796(60 \%)$ & \\
Full & 17.5 & 6331 & $2810(44 \%)$ & {$[30 \%]$} \\
Full & 18.0 & 13361 & $3931(29 \%)$ & {$[16 \%]$} \\
Full & 18.5 & 27177 & $4793(18 \%)$ & {$[6 \%]$} \\
\hline Inter-cluster & 17.0 & 2404 & $1166(49 \%)$ & \\
Inter-cluster & 17.5 & 5105 & $1693(33 \%)$ & {$[20 \%]$} \\
Inter-cluster & 18.0 & 10862 & $2285(21 \%)$ & {$[10 \%]$} \\
Inter-cluster & 18.5 & 21917 & $2887(13 \%)$ & {$[5 \%]$} \\
\hline
\end{tabular}

\section{The spherical collapse model}

We have applied the spherical collapse model (Regös \& Geller 1989) to the SSC. The resulting mass enclosed by radius $r=8 h^{-1} \mathrm{Mpc}$ lies between $2.0 \times 10^{15}$ and $1.3 \times$ $10^{16} h^{-1} M_{\odot}$, corresponding to a density range $\rho / \rho_{c} \sim 3-20$ (Reisenegger et al. 2000). Diaferio's (1999) method gives similar results for the lower limit of the virialized mass in clusters. The mass required at the distance of the SSC to produce the observed motion of the Local Group with respect to the CMB is $M_{\text {dipole }} \approx(2-5) \times 10^{17} h^{-1} M_{\odot} \Omega^{0.4}$ (Reisenegger et al. 2000, Hoffman et al. 2001). The mass within $8 h^{-1} \mathrm{Mpc}$ can therefore produce at most $\simeq 5-10 \%$ of the observed Local Group motion, which makes it unlikely that even the whole SSC would dominate its gravitational acceleration. On the other hand, consistent models of the density and velocity distribution on large scales (where density fluctuations are small) in the local Universe can now be built (e.g. Branchini et al. 1999). In these, the SSC figures prominently, although the Local Group motion originates from a combination of several "attractors".

\section{Acknowledgements}

This work was partially supported by the cooperative program ECOS/CONICYT C96U04 and the PICS-CNPq France-Brazil cooperation 910068/00-3.

\section{References}

Bardelli S., Zucca E., Baldi A.: 2001, MNRAS 320, 387.

Branchini E. et al. : 1999, MNRAS 308, 1.

Diaferio A: 1999, MNRAS 309, 610.

Drinkwater M.J., Proust D., Parker Q.A., Quintana H., Slezak E.: 1999, PASA 16, 113.

Drinkwater M.J., Parker Q.A., Proust D., Quintana H., Slezak E.: 2004, PASA 21, 89.

Hoffman Y., Eldar A., Zaroubi S., Dekel A.: 2001, astro-ph/0102190v1.

Jones H., et al. (6dFGRS): 2004, MNRAS submitted.

Kaldare R., Colless M., et al. : 2003, MNRAS 339, 652.

Proust D., Quintana H., Reisenegger A.: 2004, $A \mathscr{E} A$ in preparation.

Quintana H., Ramírez A., Melnick J., et al. : 1995, AJ 110, 463.

Quintana H., Melnick J., Proust D., Infante L.: 1997, A\&SAS 125, 247.

Quintana H., Carrasco E.R., Reisenegger, A: 2000, AJ 120, 511.

Regös E., Geller M.J.: 1989, AJ 98, 755.

Reisenegger A., Quintana H.., et al. : 2000, $A J$ 120, 523. 